\title{
Cryo-Balloon Catheter Localization in Fluoroscopic Images
}

\author{
Tanja Kurzendorfer ${ }^{a}$, Alexander Brost ${ }^{b}$, Carolin Jakob ${ }^{a}$, Philip W. \\ Mewes $^{c}$, Felix Bourier ${ }^{b, d}$, Martin Koch ${ }^{a}$, Klaus Kurzidim ${ }^{d}$, Joachim \\ Hornegger $^{a}$ and Norbert Strobel ${ }^{c}$
}

${ }^{a}$ Pattern Recognition Lab, Friedrich-Alexander-University Erlangen-Nuremberg, Erlangen, Germany

${ }^{b}$ Department of Radiology, Stanford University, Stanford, CA, USA

${ }^{c}$ Siemens AG, Healthcare Sector, Forchheim, Germany

${ }^{d}$ Klinik für Herzrhythmusstörungen, Krankenhaus Barmherzige Brüder,
Regensburg, Germany

\begin{abstract}
Minimally invasive catheter ablation has become the preferred treatment option for atrial fibrillation. Although the standard ablation procedure involves ablation points set by radio-frequency catheters, cryo-balloon catheters have even been reported to be more advantageous in certain cases. As electro-anatomical mapping systems do not support cryo-balloon ablation procedures, X-ray guidance is needed. However, current methods to provide support for cryo-balloon catheters in fluoroscopically guided ablation procedures rely heavily on manual user interaction. To improve this, we propose a first method for automatic cryo-balloon catheter localization in fluoroscopic images based on a blob detection algorithm. Our method is evaluated on 24 clinical images from 17 patients. The method successfully detected the cryoballoon in 22 out of 24 images, yielding a success rate of $91.6 \%$. The successful localization achieved an accuracy of $1.00 \mathrm{~mm} \pm 0.44 \mathrm{~mm}$. Even though our methods currently fails in $8.4 \%$ of the images available, it still offers a significant improvement over manual methods. Furthermore, detecting a landmark point along the cryo-balloon catheter can be a very important step for additional post-processing operations.
\end{abstract}

Keywords: Ablation Procedures, Atrial Fibrillation, Catheter Ablation, Catheter Localization, Cryo-Thermal, Cryo-Balloon, Electrophysiology

\section{MOTIVATION}

Atrial fibrillation is one the most common cardiac arrhythmias, currently affecting 6 million people in Europe [1]. Nowadays, atrial fibrillation is often treated by minimal catheter ablation procedures, in particular when pharmacotherapy fails $[2,3]$. Pulmonary vein isolation by minimally invasive catheter ablation has become an effective treatment approach for paroxysmal atrial fibrillation [4]. The standard technique electrically isolates the pulmonary veins with a radio-frequency ablation catheter [5]. With these catheters a point-by-point ablation is required. This technique is rather time consuming and bears certain risks, e.g., PV stenosis, tamponade, or esophageal fistula. So called single shot devices such as the cryothermal balloon catheter have been introduced to simplify the ablation of pulmonary veins and reduce procedure times as well as risks [6]. An example of a cryothermal balloon catheter is shown in Figure 1 (a). These devices try to isolate a pulmonary vein with a single application. The cryo-balloon

Send correspondence to Alexander Brost: E-mail: brost@stanford.edu 


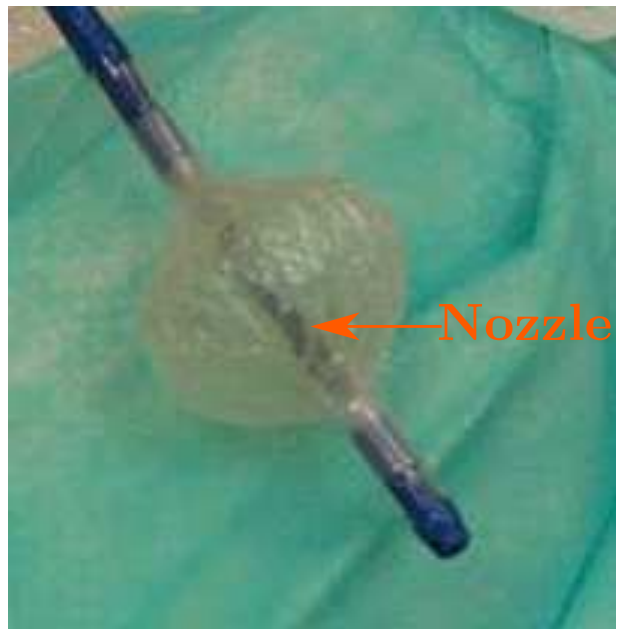

(a)

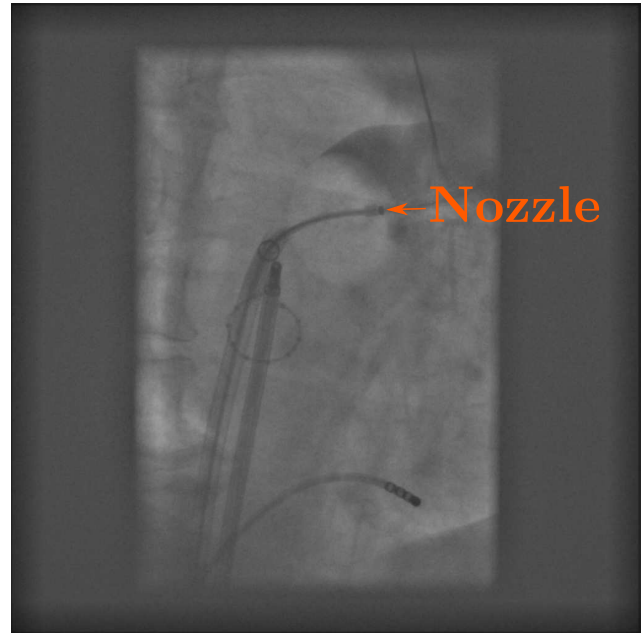

(b)

Figure 1: (a) An example of an inflated cryothermal balloon catheter. The balloon was manually inflated to its diameter of $28 \mathrm{~mm}$. Courtesy of Alexander Brost. (b) Original fluoroscopic image with indicated nozzle (orange).

catheter is pressed against the PV antrum before it gets filled with liquid nitrogen which, upon vaporization, freezes the tissue around the PV electrically isolating the left atrium [7].

Ablation procedures are performed in electrophysiology labs equipped with modern C-Arm $\mathrm{X}$-ray systems, that provide the possibility to overlay pre-operative data onto the live fluoroscopic images [8-10]. In addition, electro-anatomical mapping systems may be used as well [11]. As of now, there is no mapping system that offers support for cryo-balloon ablation procedures, as they do not carry any electrodes or position sensors $[12,13]$. Recent developments utilize image processing techniques to facilitate cryo-balloon navigation involving augmented fluoroscopy techniques $[12,14,15]$. These methods still need a high degree of manual interaction. To improve the situation, we propose a method for automatic cryo-balloon localization in fluoroscopic images based on blob detection to improve image guidance provided by fluoroscopic systems.

\section{METHODS}

The cryo-balloon itself is hard to see in fluoroscopic images, even for the human eye. The injection tube attached to the cryo-balloon catheter on the other hand is easier to see. During a cardiac ablation procedure, pressurized liquid nitrous oxide travels to the injection tube - or nozzle - of the cryo-balloon catheter. There, it is sprayed into the balloon. As it vaporizes, it absorbs heat from the surrounding tissue, thereby cooling and freezing the target tissue. We consider the nozzle as the best surrogate to detect the cryo-balloon catheter, see Figure 1 (a) for an example.

Our algorithm is a filter based approach, performed in following steps. First, the collimators are removed. Collimators or shutters are often used to reduce radiation. Once the collimators have been removed, the image processing steps afterwards are focused only on the region of interest. An example of the cropped image is given in Figure 2 (b). After shutter removal, we have the cropped image, denoted by $\mathbf{I}(u, v) \in \mathbb{Z}^{N \times M}$ of size $N \times M$. Then a histogram equalization is performed. This step is used to enhance the difference between the cryo-balloon catheter nozzle and the surrounding tissue [7]. The histogram equalization is applied to the cropped image only. An example of the image $\mathbf{I}_{h}(u, v)$ after histogram equalization is depicted in Figure 2 (c). In the next step, a blob detection algorithm is applied to the histogram-equalized image 


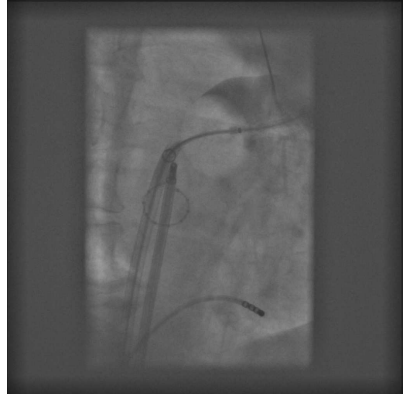

(a)

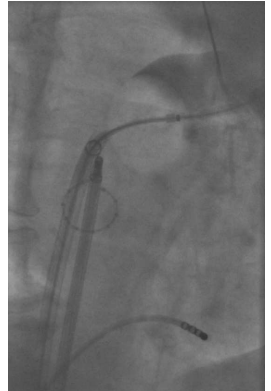

(b)

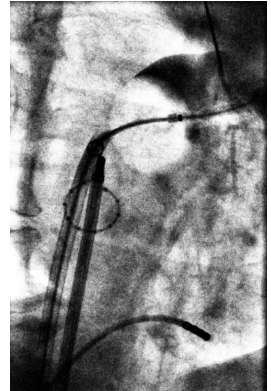

(c)

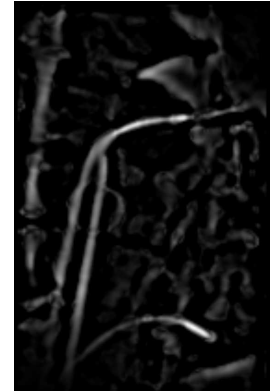

(d)

Figure 2: (a) Original fluoroscopic image of size 1,024 $\times 1,024$ pixels. (b) Cropped image after collimator removal. (c) Cropped image after histogram equalization. (d) Image after filtering with second-order derivatives of the Gaussian.

$\mathbf{I}_{h}(u, v)$. The blob detector is based on the Hessian matrix [16]. We do not use a multi-scale blob detector, because the nozzle of the cryo-balloon catheter has always the same dimensions, because there is currently only one manufacturer of cry-balloons who produces devices that differ in size only. The second derivatives of a Gaussian function are

$$
\begin{aligned}
\mathbf{G}_{u u}(u, v) & =-\frac{1}{2 \pi \sigma^{4}}\left(1-\frac{u^{2}}{\sigma^{2}}\right) e^{\frac{-\left(u^{2}+v^{2}\right)}{2 \sigma^{2}}} \\
\mathbf{G}_{v v}(u, v) & =-\frac{1}{2 \pi \sigma^{4}}\left(1-\frac{v^{2}}{\sigma^{2}}\right) e^{\frac{-\left(u^{2}+v^{2}\right)}{2 \sigma^{2}}} \\
\mathbf{G}_{u v}(u, v) & =-\frac{u v}{2 \pi \sigma^{4}} e^{\frac{-\left(u^{2}+v^{2}\right)}{2 \sigma^{2}}} .
\end{aligned}
$$

Due to the isotropic filter kernel, the second derivatives $\mathbf{G}_{u v}$ and $\mathbf{G}_{v u}$ are equal. The standard deviation $\sigma$ of the Gaussian filter kernel represents the diameter of the cryo-balloon catheter nozzle to be detected. As the thickness of the catheter is currently known and the pixel spacing is available either from DICOM information or C-Arm system parameters, there is no need to vary the standard deviation $\sigma$ of the Gaussian. When the image $\mathbf{I}_{h}(u, v)$ is convolved with the second order Gaussian derivative, for example with $\mathbf{G}_{u u}(u, v)$, we get $\mathbf{I}_{u u}(u, v)$ as

$$
\mathbf{I}_{u u}(u, v)=\mathbf{I}_{h}(u, v) * \mathbf{G}_{u u}(u, v),
$$

where $*$ denotes the convolution operator. Similar definitions apply for $\mathbf{I}_{v v}(u, v)$, and $\mathbf{I}_{u v}(u, v)$. Then the eigenvalues $\eta_{1,2}(u, v) \in \mathbb{R}$ of the Hessian matrix $\mathbf{H}(u, v) \in \mathbb{R}^{2 \times 2}$ are calculated. For the blob detection, we look for the highest eigenvalues and define them as candidates for the cryo-balloon catheter nozzle. Currently, the 300 strongest blobs $\mathbf{b}_{i}=\left(u_{i}, v_{i}\right)$ are considered as candidates for the cryo-balloon catheter,

$$
\mathcal{B}_{0}=\left\{\mathbf{b}_{i} \mid \mathbf{V}\left(u_{1}, v_{1}\right) \geq \mathbf{V}\left(u_{i}, v_{i}\right) \geq \mathbf{V}\left(u_{300}, v_{300}\right)\right\}
$$

where $\mathbf{b}_{i}$ denotes the $\mathrm{i}^{\text {th }}$ blob, see Figure 3 (a) for an example. From this set, the nozzle is detected using the following different criteria:

- Blobs within a certain margin to the image boundary are excluded. This leads to a subset of nozzle candidates described by

$$
\mathcal{B}_{1}=\left\{\mathbf{b}_{i} \mid \mathbf{b}_{i} \in \mathcal{B}_{0} \wedge\left(\theta_{u l}<u_{i}<\theta_{u u}\right) \wedge\left(\theta_{v l}<v_{i}<\theta_{v u}\right)\right\}
$$

where $\theta_{u l}$ is the lower limit for $u$ and $\theta_{u u}$ is the upper limit. Respectively, $\theta_{v l}$ is the lower limit for $v$ and $\theta_{v u}$ is the upper limit, see Figure 3 (b) for an example. 


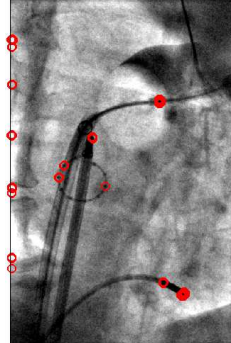

(a)

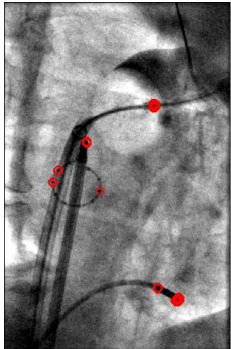

(b)

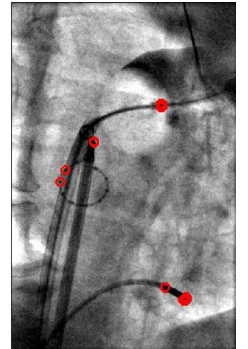

(c)

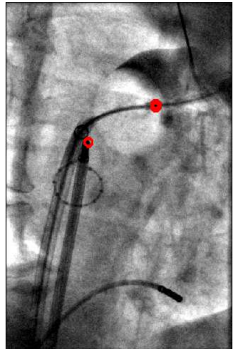

(d)

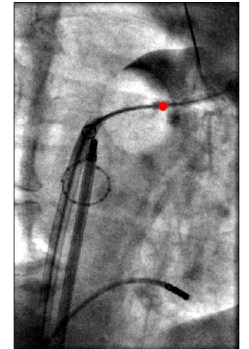

(e)

Figure 3: Blob selection pipeline. (a) Strongest blobs (max. 300). (b) Blobs removed within a certain margin to the image boundary. (c) Without outliers. (d) Without blobs that are close to other blobs. (e) Final cryo-balloon catheter nozzle.

- Blobs that occur scarcely in a certain distance are excluded. This further reduces the set of blobs to

$$
\mathcal{B}_{2}=\left\{\mathbf{b}_{i} \mid \mathbf{b}_{i} \in \mathcal{B}_{1} \backslash\left(\left\|\mathbf{b}_{i}-\mathbf{b}_{j}\right\|_{2}<\theta_{z}, \forall \mathbf{b}_{j} \in \mathcal{B}_{1}\right) \leq \theta_{c}\right\},
$$

where $\theta_{z}$ is the distance between two blobs and $\theta_{c}$ is the threshold for the number of occurrences of certain blobs in the region defined by $\theta_{z}$. An example of this exclusion is given in Figure 3 (c).

- Blobs that are close to other blobs are excluded, as the nozzle of the cryo-balloon is isolated because once the cryo-balloon is inflated, no other catheter can be placed close to it. By doing so, the coronary sinus and the circumferential mapping catheter can be excluded. The remaining blob candidates are given by the following set,

$$
\mathcal{B}_{3}=\mathcal{B}_{3}=\left\{\mathbf{b}_{i} \mid \mathbf{b}_{i} \in \mathcal{B}_{2} \backslash\left(\theta_{u}>\left\|\mathbf{b}_{i}-\mathbf{b}_{j}\right\|_{2}>\theta_{l}\right), \forall \mathbf{b}_{j} \in \mathcal{B}_{2}\right\}
$$

where $\theta_{l}$ denotes the lower limit and $\theta_{l}$ the upper limit. See Figure 3 (d) for an example.

- Blobs within a brighter area with a small dark region around the blob are preferred. When the cryo-balloon is inflated, liquid nitrogen replaces the blood in the vicinity of the nozzle. The brighter region around a blob $\mathbf{b}_{i}$ is calculated as follows,

$$
m_{h}\left(\mathbf{b}_{i}\right)=\sum_{u=b_{u}-\theta_{1}}^{b_{u}+\theta_{1}} \sum_{v=b_{v}-\theta_{1}}^{b_{v}+\theta_{1}} \mathbf{I}_{h}(u, v) .
$$

The darker region is smaller, as it covers only the tip of the cryo-balloon catheter. It is calculated as follows,

$$
m_{d}\left(\mathbf{b}_{i}\right)=\sum_{u=b_{u}-\theta_{2}}^{b_{u}+\theta_{2}} \sum_{v=b_{v}-\theta_{2}}^{b_{v}+\theta_{2}} \mathbf{I}_{h}(u, v) .
$$

Given $m_{h}\left(\mathbf{b}_{i}\right)$ and $m_{d}\left(\mathbf{b}_{i}\right)$, the final estimate for the catheter nozzle $\hat{\mathbf{b}}$ can be computed, by considering the maximum of the subtraction $m_{d}\left(\mathbf{b}_{i}\right)$ from $m_{h}\left(\mathbf{b}_{i}\right)$,

$$
\hat{\mathbf{b}}=\arg \max _{b_{i} \in \mathcal{B}_{3}}\left(m_{h}\left(\mathbf{b}_{i}\right)-m_{d}\left(\mathbf{b}_{j}\right)\right) .
$$

Finally, the detected cryo-balloon catheter nozzle is given as $\hat{\mathbf{b}}$, see Figure 3 (e). 


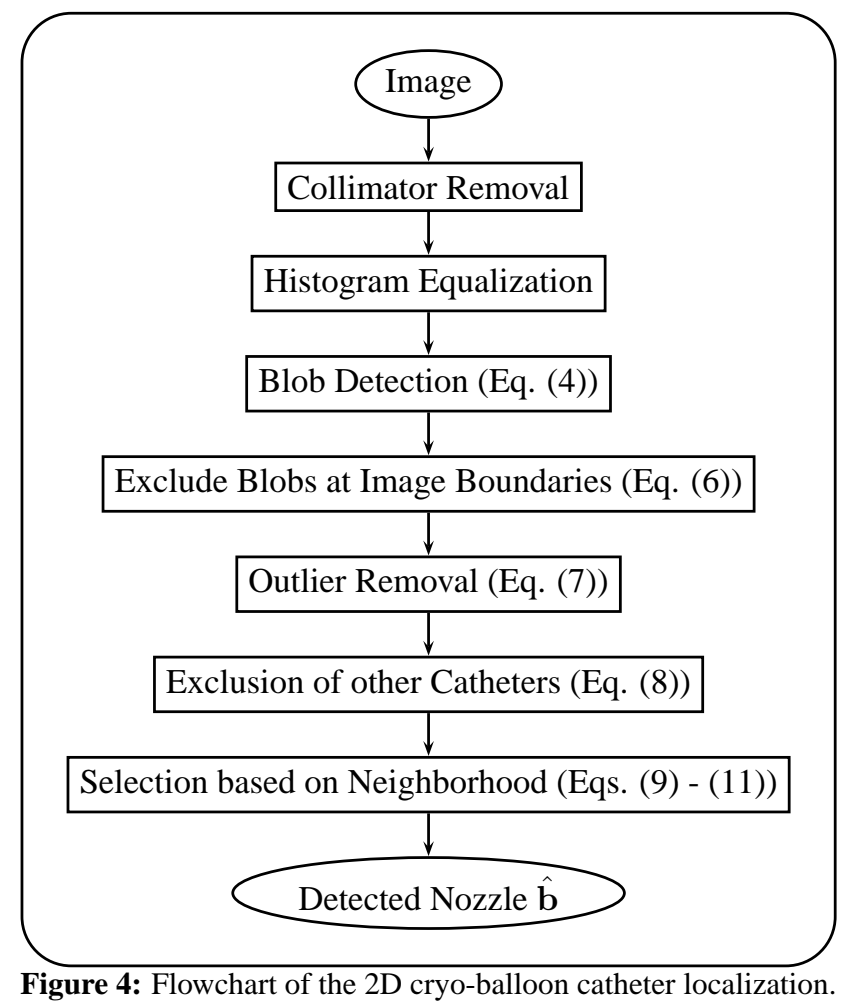

\section{EVALUATION AND RESULTS}

For the localization of the cryo-balloon catheter nozzle, several parameters need to be specified. The first parameter is the standard deviation of the Gaussian $\sigma$, representing the size of the cryo-balloon catheter nozzle. As this is fixed and the pixel spacing is available, there is no need to vary $\sigma$. In all clinical data sets the pixel spacing was $0.1725 \mathrm{~mm}$ per pixel.

For the blob selection, several thresholds are required. Currently, we consider the 300 strongest blobs $\mathbf{b}_{i}$. This parameter is heuristically chosen. The next exclusion criteria is the distance to the image boundaries $\theta_{u l}, \theta_{u u}, \theta_{v l}$ and $\theta_{v u}$. Based on the available data sets, a margin of 22 pixels with respect to the image boundaries was used. The outlier exclusion requires two parameters, the distance between two blobs $\theta_{z}$ and the limit for occurrence $\theta_{c}$ of blobs in a certain distance. Those parameters were chosen as 10 pixels for $\theta_{z}$ and 3 blobs for $\theta_{c}$. To exclude blobs that are close to other blobs, two further thresholds $\theta_{u}$ and $\theta_{l}$ were used and chosen to be 62 pixels and 30 pixels, respectively. The final cryo-balloon catheter nozzle estimation was performed with the help of the cost function Eq. (11). As the blobs are within a brighter area and a small dark region, the size of the bright area was chosen to be $39 \times 39$ pixels, where $\theta_{1}$ is set to 19 pixels. For the small dark region, we have chosen 3 pixels for $\theta_{2}$. These thresholds were determined by considering all available data. Our method was evaluated on 24 clinical fluoroscopic images from 17 patients. The sequences were acquired at one clinical site during a regular procedure, stored, anonymized, and then evaluated during a post-processing step. Since the images were taken during standard EP procedures, they show one circumferential mapping catheter, one catheter in the coronary sinus and a cryo-balloon catheter as well. In addition to that, some images also display ECG leads that were attached to the skin of the patient.

For the evaluation, a physician was asked to pick the center of the cryo-thermal balloon nozzle. We took this as the the reference position denoted as $\mathbf{b}^{\star}$. The detected 2-D position of the cryo-balloon catheter nozzle is denoted as $\hat{\mathbf{b}}$. The detection error $\epsilon$ is calculated as the 


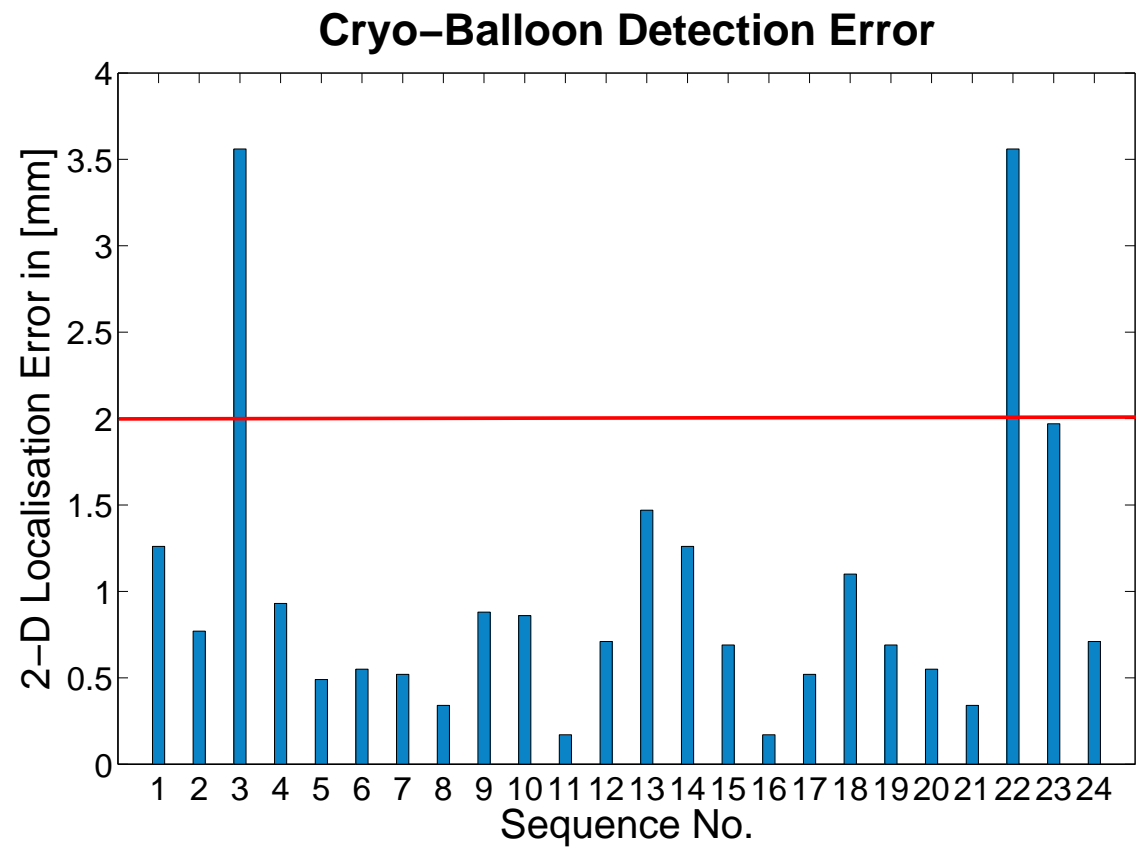

Figure 5: Localization accuracy of the proposed cryo-balloon catheter localization method. Our approach achieves an accuracy of $1.00 \mathrm{~mm} \pm 0.89 \mathrm{~mm}$. The smallest error was $0.17 \mathrm{~mm}$. The largest error was 3.56 $\mathrm{mm}$.

Euclidean distance between the detected point and the corresponding reference point

$$
\epsilon=\left\|\mathbf{b}^{\star}-\hat{\mathbf{b}}\right\|_{2}
$$

Our proposed method achieved an overall detection accuracy of $1.00 \mathrm{~mm} \pm 0.89 \mathrm{~mm}$ with a total minimum of $0.17 \mathrm{~mm}$ and a total maximum of $3.56 \mathrm{~mm}$. We considered a localization failure if the detected point was $2.00 \mathrm{~mm}$ away from the actual gold-standard annotation. The detection errors are given in Figure 5. From this, we see that our method fails in two sequences. We attribute a large part of the error to the fact, that our gold-standard annotation is reduced to a single point and the nozzle of the cryo-balloon has a size of about $2.6 \mathrm{~mm}$.

\section{DISCUSSION AND CONCLUSIONS}

Our proposed method successfully detected a cryo-thermal balloon catheter in all 22 out of 24 clinical sequences, yielding a successful localization rate of $91.6 \%$. In those cases, the accuracy of $0.77 \mathrm{~mm} \pm 0.44 \mathrm{~mm}$ is below the clinical acceptable threshold of $2.00 \mathrm{~mm}$. A comparison between our 22 successful localization results and a gold-standard annotation is given in Figure 6. Even though our methods currently fails in $8.4 \%$ of the images available, it succeeds much more often than it fails, thus providing valuable assistance for a detection task that is otherwise completely manual. Although the gold-standard annotation is from a clinical expert, it still involves inaccuracies regarding the manual selection of the catheter nozzle. In sum, this work demonstrates that cryo-balloon catheter detection and localization using a simple blob detection is possible.

While the information obtained from a single detected point itself may not directly help the physician during his procedure, our result will enable other methods, e.g. automatic cryoballoon reconstruction from two views. The use of a classifier might also further improve the detection [17]. 


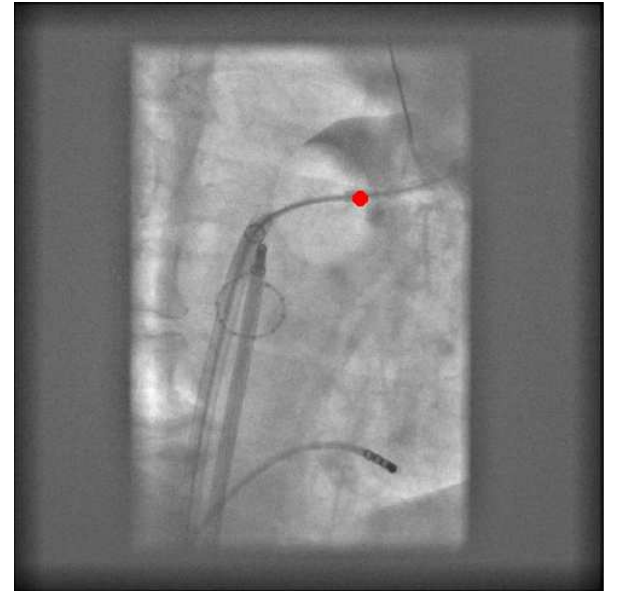

(a)

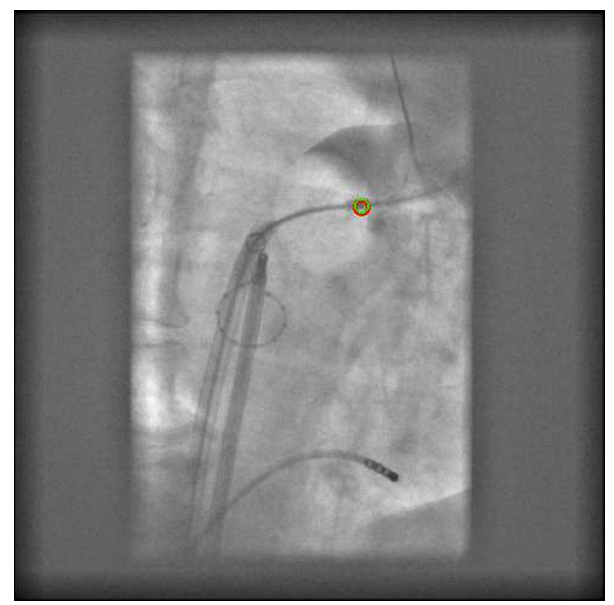

(b)

Figure 6: (a) Result of our cryo-balloon catheter localization. (b) The same image with our localization result (red) and the gold-standard annotation (green).

\section{Acknowledgements}

This work has been supported by the German Federal Ministry of Education and Research (BMBF), project grant No. 01EX1012E, in the context of the initiative Spitzencluster Medical Valley - Europäische Metropolregion Nürnberg. Additional funding was provided by Siemens AG, Healthcare Sector.

\section{References}

[1] A. Camm, P. Kirchhof, G. Lip, U. Schotten, I. Savelieva, S. Ernst, I. van Gelder, N. AlAttar, G. Hindricks, B. Prendergast, H. Heidbüchel, O. Alfieri, A. Angelini, D. Atar, P. Colonna, R. de Caterina, J. de Sutter, A. Goette, B. Gorenek, M. Heldal, S. Hohloser, P. Kolh, J. L. Heuzey, P. Ponikowski, and F. Rutten, "Guidelines for the management of atrial fibrillation: The Task Force for the Management of Atrial Fibrillation of the European Society of Cardiology (ESC)," European Heart Journal 31, 2369-2429 (2010).

[2] A. Brost, F. Bourier, A. Wimmer, M. Koch, A. Kiraly, R. Liao, K. Kurzidim, J. Hornegger, and N. Strobel, "Real-time circumferential mapping catheter tracking for motion compensation in atrial fibrillation ablation procedures," in Proceedings of SPIE Medical Imaging 2012: Image-Guided Procedures, Robotic Interventions, and Modeling, San Diego, CA, USA, Febuary 4-9, D. Holmes and K. Wong, Eds., 8316, 83162V (2012).

[3] S. Kaeppler, A. Brost, W. Wu, M. Koch, F. Bourier, T. Chen, M. Ostermeier, K. Kurzidim, J. Hornegger, and N. Strobel, "Motion Estimation Model for Cardiac and Respiratory Motion Compensation," in 3rd International Conference on Information Processing in Computer-Assisted Interventions (IPCAI 2012), Pisa, Italy, June 27, 94-104 (2012).

[4] Calkins, H., Brugada, J., et al., "HRS/EHRA/ECAS Expert Consensus Statement on Catheter and Surgical Ablation of Atrial Fibrillation: Recommendations for Personnel, Policy, Procedures and Follow-Up," Heart Rhythm 4, 1-46 (2007).

[5] A. Kleinoeder, A. Brost, F. Bourier, M. Koch, K. Kurzidim, J. Hornegger, and N. Strobel, "Cryo-balloon catheter position planning using AFiT," in Proceedings of SPIE Medical Imaging 2012: Image-Guided Procedures, Robotic Interventions, and Modeling, San Diego, CA, USA, Febuary 4-9, D. Holmes and K. Wong, Eds., 8316, 83162R (2012).

[6] M. Schneider, M. K. Schade, and B. Schumacher, "Cryoballoon ablation of paroxysmal atrial fibrillation within the dilated coronary sinus in a case of persistent left superior vena cava," Europace 11, 1387-1389 (2009). 
[7] A. Brost, Image Processing for Fluoroscopy Guided Atrial Fibrillation Ablation Procedures. PhD thesis, Friedrich-Alexander-Universität (2012).

[8] F. Bourier, D. Vukajlovic, A. Brost, J. Hornegger, N. Strobel, and K. Kurzidim, "Pulmonary vein isolation supported by mri-derived 3d-augmented biplane fluoroscopy: A feasibility study and a quantitative analysis of the accuracy of the technique," Journal of Cardiovascular Electrophysiology (2012).

[9] A. Brost, A. Wimmer, R. Liao, J. Hornegger, and N. Strobel, "Constrained 2-d/3-d registration for motion compensation in afib ablation procedures," in Information Processing in Computer-Assisted Interventions, R. Taylor and G.-Z. Yang, Eds., Lecture Notes in Computer Science 6689, 133-144, Springer Berlin / Heidelberg (2011).

[10] A. Brost, A. Wimmer, F. Bourier, M. Koch, R. Liao, K. Kurzidim, N. Strobel, and J. Hornegger, "Constrained Registration for Motion Compensation in Atrial Fibrillation Ablation Procedures ," IEEE Transactions on Medical Imaging [Epub ahead of print], [n/a] (2011).

[11] L. Gepstein, G. Hayam, and S. Ben-Haim, "A Novel Method for Nonfluoroscopic Catheter-Based Electroanatomical Mapping of the Heart: In Vitro and In Vivo Accuracy Results," Circulation 95, 1611-1622 (1997).

[12] F. Bourier, A. Brost, A. Kleinoeder, T. Kurzendorfer, M. Koch, A. Kiraly, H.-J. Schneider, J. Hornegger, N. Strobel, and K. Kurzidim, "Navigation for Fluoroscopy-Guided Cryo-Balloon Ablation Procedures of Atrial Fibrillation," in Proceedings of SPIE Medical Imaging 2012: Image-Guided Procedures, Robotic Interventions, and Modeling, San Diego, CA, USA, Febuary 4-9, D. Holmes and K. Wong, Eds., 8316, 831627 (2012).

[13] F. Bourier, D. Vukajlovic, H. Schneider, A. Brost, N. Strobel, J. Hornegger, and K. Kurzidim, "3D-Visualization of Cryo Balloon Catheters and the Left Atrium in Biplane Fluoroscopy to support Pulmonary Vein Isolation," in 33rd Annual Scientific Meeting, H. R. Society, Ed., 04-117 (2012).

[14] A. Kleinoeder, A. Brost, F. Bourier, M. Koch, K. Kurzidim, J. Hornegger, and N. Strobel, "Cryo-balloon reconstruction from two views," in 18th IEEE International Conference on Image Processing (ICIP 2011), Brussels, Belgium, September 11-14, 223-230 (2011).

[15] T. Kurzendorfer, A. Brost, F. Bourier, M. Koch, K. Kurzidim, J. Hornegger, and N. Strobel, "Cryo-Balloon Catheter Tracking in AtrialFibrillation Ablation Procedures," in Bildverarbeitung für die Medizin (BVM 2012), Berlin, Germany, March 18-20, 386-391 (2012).

[16] Y. Ma, A. King, N. Gogin, C. Rinaldi, J. Gill, R. Razavi, and K. Rhode, "Real-Time Respiratory Motion Correction for Cardiac Electrophysiology Procedures Using ImageBased Coronary Sinus Catheter Tracking," in MICCAI 2010, Bejing, China, Lect. Notes Comput. Sci. 6361, 391-399, Springer Berlin / Heidelberg (2010).

[17] A. Brost, A. Wimmer, R. Liao, J. Hornegger, and N. Strobel, "Catheter Tracking: FilterBased vs. Learning-Based," in Pattern Recognition, M. Goesele, S. Roth, A. Kuijper, B. Schiele, and K. Schindler, Eds., Lecture Notes in Computer Science 6376, 293-302, Springer Berlin / Heidelberg (2010). 